\title{
Adherencia a guías de atención de control prenatal en red pública de subregión norte, departamento del Magdalena (Colombia)
}

\section{Adherence to guidelines for prenatal care in public health care network of the north subregion, department of Magdalena (Colombia)}

Título corto: Adherencia a guías de atención de control prenatal

\begin{abstract}
Katty Johana Ortiz-Grisolle1 (D), Kelly Garcia-Galezo² (D), Angela Patricia Gómez-Cardona ${ }^{3}$ (D), Sindy Julieth Maestre-Álvarez ${ }^{4}$ (D), Graciela Emilia Vargas-Guerrero ${ }^{5}$ (D), Néstor Ramos-Navas ${ }^{6}$ (D), Wilson Giovanni Jiménez-Barbosa ${ }^{7}$ (i)
\end{abstract}

Tipología: Artículo de investigación científica y tecnológica

Para citar este artículo: Ortiz-Grisolle K, Garcia-Galezo K, Gómez-Cardona AP, Maestre-Álvarez SJ, VargasGuerrero V, Ramos-Navas N, et al. Adherencia a guías de atención de control prenatal en red pública de subregión norte, departamento del Magdalena (Colombia). Duazary. 2019 enero; 16(1): 115-127. Doi: http://dx.doi. org/10.21676/2389783X.2511

Recibido en octubre 23 de 2017

Aceptado en mayo 25 de 2018

Publicado en línea en septiembre 01 de 2018

\section{RESUMEN}

El objetivo del estudio fue determinar el grado de adherencia a la guía de atención prenatal en las Empresas Sociales del Estado (ESE) de primer nivel de complejidad de la subregión norte, departamento del Magdalena (Colombia) de enero a marzo de 2016. Se realizó un estudio descriptivo con revisión documental, retrospectivo, que se desarrolló en el primer trimestre del año 2016. El censo de la población estuvo representada por 1164 historias clínicas de Control Prenatal en las instituciones hospitalarias adscritas a las ESE; de las cuales se escogieron aleatoriamente 396 para la construcción de la investigación. En los resultados se evidenció la no adherencia a las guías de atención prenatal, y se observó que solo el hospital dos cumple al 100\% optimo con cuatro 66,6\%) de los seis ítems evaluados (Clasificación

1. Clinic Smile. Santa Marta, Colombia. Correo: kattyjohhana0585@gmail.com - http://orcid.org/0000-0002-7111-2937

2. Universidad Jorge Tadeo Lozano. Santa Marta, Colombia. Correo: kellygarciag@gmail.com - http://orcid.org/0000-0001-5128-0661

3. Clínica Cehoca. Santa Marta, Colombia. Correo: anpagoca14@gmail.com - http://orcid.org/0000-0002-4857-0833

4.UniversidadJorgeTadeoLozano.SantaMarta,Colombia.Correo:julietalvarez03@hotmail.com-https://orcid.org/0000-0002-0735-9978.

5. Santa Marta, Colombia. Correo electrónico: lagerente2008@gmail.com - https://orcid.org/0000-0003-1131-0138

6. Universidad de Jorge Tadeo Lozano. Santa Marta, Colombia. Correo: nestorr.ramosn@utadeo.edu.co - https://orcid. org/0000-0002-2285-2306

7. Universidad de Jorge Tadeo Lozano. Santa Marta, Colombia. Correo: wilsong.jimenezb@utadeo.edu.co - https://orcid. org/0000-0002-0467-0365 
del riesgo obstétrico, historia clínica materno y carné materno perinatal, provisión de micronutrientes y referencia y contrarreferencia). Se comprobó que se requiere mejorar el seguimiento a las alteraciones detectadas durante el embarazo, especialmente en lo que concierne a la clasificación de riesgo con el objetivo de disminuir la tasa de mortalidad materna en el departamento del Magdalena.

Palabras clave: atención prenatal; riesgo; mortalidad.

\section{ABSTRACT}

The objective of the study was to determine the degree of adherence to the prenatal care guide in the State Social Enterprises of the first level of complexity of the northern sub-region, department of Magdalena (Colombia), from January to March of 2016. A descriptive study was carried out with a retrospective documentary review, which was developed in the first quarter of 2016. The census of the population was represented by 1164 Prenatal Control clinical records in the hospitals affiliated to the State Social Enterprises; of which 396 were randomly chosen for the construction of the research. The results showed non-adherence to prenatal care guidelines, observing that only hospital two meets $100 \%$ OPTIMO with four $66.6 \%$ ) of the six items evaluated (Classification of obstetric risk, maternal clinical history and maternal cardiology perinatal, provision of micronutrients and reference and counterreferral). It was found that it is necessary to improve the monitoring of the alterations detected during pregnancy, especially as regards the risk classification with the aim of decreasing the maternal mortality rate in the department of Magdalena.

Keywords: Prenatal Care; Risk; Mortality.

\section{INTRODUCCIÓN}

$\mathrm{L}^{2}$ a Organización Mundial de la Salud (OMS) ${ }^{1}$ considera que un Control Prenatal (CPN) de buena calidad es aquel que inicia en el primer trimestre del embarazo, cuando se educa, protege, detecta y se hace tratamiento de enfermedades que aparecen durante la gestación. Según la misma $\mathrm{OMS}^{1}$, la atención de salud profesional durante el embarazo, el parto y el período posnatal (inmediatamente posterior al parto) evita complicaciones a la madre y al recién nacido, y permite la detección y tratamiento tempranos de problemas de salud. En el mundo, cerca del $80 \%$ de las mujeres son asistidas por lo menos una vez durante el embarazo por personal de salud capacitado; en países de ingreso medio alto, la cobertura es del $95 \%{ }^{2}$.

En Colombia, según la Encuesta Nacional de Demografía y Salud (ENDS), “para 2010, el 91,7\% de las mujeres encuestadas que habían dado a luz en los cinco años previos a la encuesta recibieron atención prenatal de un profesional médico, lo cual representa un 4,6\% más que lo encontrado en la ENDS 20053; así mismo, el $77 \%$ tuvo el primer control prenatal antes del cuarto mes de embarazo, lo cual significa un incremento de $6,5 \%$ con respecto al 2005; el 15\% lo tuvo entre el cuarto y el quinto mes, y el restante $8 \%$ lo tuvo pasados los cinco meses" 2 . De acuerdo con los registros de estadísticas vitales, “entre 2005 y 2013 el 83,45\% de los nacidos vivos tuvieron cuatro o más controles prenatales; el promedio para cada neonato fue de seis consultas. En la mayoría de los departamentos, más del $80 \%$ de los recién nacidos tuvieron cuatro o más controles prenatales, manteniendo el promedio de estos controles entre 5 y 7 "2.

Sin embargo, a pesar de estos avances en el acceso a servicios de salud de atención prenatal, la mortalidad materna se ha convertido en un problema de salud pública, derivado no solo de la provisión y acceso a infraestructuras y proce- 
sos de atención en salud, sino también ligado a la percepción sobre la naturaleza de la mujer, su subordinación genérica y a prácticas personales, comunitarias y sociales que aumentan los riesgos obstétricos y obstaculizan la implementación de políticas de control prenatal ${ }^{3}$.

Por este grado de complejidad y su trascendencia para el desarrollo de los países; el bienestar de sus poblaciones, reducir la mortalidad materna y lograr el acceso universal a la salud reproductiva fueron incluidos dentro de los Objetivos de Desarrollo del Milenio (ODM) a ser cumplidos por los Estados miembros de la Naciones Unidas. Sin embargo, al evaluar el avance en el cumplimiento de este objetivo se observó a nivel mundial "un avance lento y desigual, en particular en los sectores más pobres de la población y para los habitantes de zonas rurales, tanto en cada país como entre países. Igualmente se registró un elevado número de muertes prevenibles asociadas a la maternidad, lo que sigue siendo inaceptable, ya que, en especial, las adolescentes son las que se enfrentan a mayores riesgos"2.

En Colombia, Según el Departamento Administrativo Nacional de Estadística DANE, en el 2013 la mortalidad materna fue de 54,62 por cada 100.000 nacidos vivos, cifra que para el 2014 se ubicó en 53,7 muertes por cada 100.000 nacidos vivos, lo que conllevó a que el país superara el estimado de 45 fallecidas por cada 100.000 nacidos vivos dentro de los compromisos de los ODM, situación que evidencia que, si bien se han venido dando avances, persisten grandes retos a nivel nacional en cuanto a esta problemática ${ }^{4}$.

Existe consenso en relación con los factores asociados a la muerte materna, en el sentido de que es un evento no siempre predecible, no siempre intervenible y en su ocurrencia se conjugan: primero, una combinación de elementos biológicos y sociales; segundo, la configuración de urgencias médicas y tercero, la confluencia de un conjunto de factores originados en la estructura socioeconómica y demográfica de cada territorio ${ }^{5,6}$.

Más específicamente, los riesgos relacionados frecuentemente con la morbilidad y mortalidad materna son la edad, la multiparidad, la anemia, el estado nutricional, los antecedentes de enfermedades crónicas como la diabetes y la hipertensión, el trabajo de parto prolongado y la cesárea. Los anteriores factores derivan en Morbilidad Materna Extremadamente Grave (MMEG), definida como la complicación grave que ocurre durante el embarazo, el parto y/o el puerperio, que pone en riesgo la vida de la mujer y requiere de una atención inmediata con el fin de evitar la muerte ${ }^{7,8}$.

Para ello, la aplicación del enfoque de riesgo precisa el uso de un plan de manejo individual y personalizado para cada gestante, dependiendo del diagnóstico y del nivel de riesgo del embarazo9. Con tal propósito, el Estado colombiano ha definido un conjunto de estándares con recomendaciones asistenciales que intentan proporcionar a las instituciones, pacientes, familiares, investigadores, administradores de planes de beneficios y demás personas interesadas, información acerca de los aspectos relacionados con la detección temprana de alteraciones del embarazo ${ }^{10}$.

Así, de acuerdo con la resolución 412 de $2000^{11}$, se entiende como valoración del riesgo materno fetal a: "la identificación de los factores de riesgo biosicosocial que afectan a una mujer en edad fértil, embarazada o en posparto y que son clasificados para determinar el grado de complejidad de la patología que presenta y el nivel de atención al que le corresponde brindar el servicio", y a las guías de atención como: [...] el documento mediante el cual se establecen las actividades, procedimientos e intervenciones a seguir y el orden secuencial y lógico para el adecuado diag- 
nóstico y tratamiento de las enfermedades de interés en salud pública [...]" ${ }^{11}$.

Así, las guías de atención clínica de control prenatal son instrumentos desarrollados sistemáticamente para ayudar a clínicos y pacientes a tomar decisiones apropiadas sobre una o varias circunstancias clínicas y para evaluar la calidad de los servicios de salud; además, pueden orientar procesos de atención de eventos específicos, contribuyendo a la disminución de la variabilidad en las prácticas y facilitando la racionalización en los costos de la provisión de los servicios de salud ${ }^{12}$.

Con las guías de atención se pretende hacer mejoras y tomar acciones que permitan llevar buenas prácticas clínicas por parte de los profesionales, y que los pacientes participen activamente dando su punto de vista y expresando sus preferencias y expectativas acerca de los servicios de salud ${ }^{13,14}$. La implementación de las guías muestra la aceptación de los profesionales de la salud con respecto al manejo estandarizado de las diferentes patologías médicas. Debido a lo anterior, se puede inferir que una adecuada adherencia se refleja en procesos diseñados, estandarizados y que puedan ser medidos por indicadores de gestión ${ }^{15}$.

Se comprende por adherencia a la capacidad y disposición del proveedor de servicios y del paciente para llegar a un acuerdo y compromiso en relación con las recomendaciones de salud pertinentes ${ }^{16}$. Para esta investigación, la adherencia fue evaluada respecto al grado de cumplimiento que el equipo humano perteneciente al prestador de servicios de salud tuvo respecto a seguir los procesos de atención definidos en las guías de atención de control prenatal establecidas en la ya mencionada Resolución 412 de $2000^{11}$ y sus anexos técnicos. Dicha evaluación se llevó a cabo en la red de prestación de servicios de salud del departamento del Magdalena - Colombia, subregión norte.
La red de prestación de servicios de salud del departamento del Magdalena está conformada por cinco subregiones: Santa Marta, Norte, Centro, Río y Sur, las cuales agrupan a los 29 municipios y al Distrito Turístico, Cultural e Histórico de Santa Marta. En la subregión norte, objeto de este estudio, se encuentran los municipios de Ciénaga, Zona Bananera, Pueblo Viejo, El Retén, Aracataca, Fundación y Algarrobo, con una población total de 376.382 habitantes. Para la atención de esta población, la subregión norte cuenta con dos instituciones de salud de mediana complejidad, el Hospital San Cristóbal de Ciénaga y San Rafael de Fundación, y seis instituciones de salud de baja complejidad de atención ${ }^{17}$.

En todas las subregiones en los últimos cinco años se ha venido realizando la evaluación del desempeño de la gestión gerencial en las ESE, en el marco de lo establecido en la Resolución 743 de 2013, y se ha detectado falta de adherencia en la prescripción de al menos un paraclínico en el primer trimestre de la gestación, y fallas en la adherencia a las Guías de Práctica Clínica en los criterios de remisión de las gestantes a asesoría preparto, cita con odontología y educación ${ }^{18}$.

Por lo hasta aquí descrito, la presente investigación se planteó como objetivo general: determinar el grado de adherencia a la guía de atención prenatal en las instituciones de salud de primer nivel de complejidad de la red pública prestadora de servicios de salud del departamento del Magdalena, en el periodo comprendido entre enero y marzo de 2016.

\section{MATERIALES Y MÉTODOS}

\section{Tipo de estudio}

Estudio cuantitativo, descriptivo, con revisión documental, retrospectiva, desarrollado en el 
primer trimestre del año 2016, a partir de la base de datos de historias clínicas suministrada por la Secretaría de Salud del Departamento del Magdalena de pacientes maternas de las instituciones de salud de cada uno de los municipios que hacen parte de la subregión norte.

\section{Población y muestra}

La población constó de 1164 historias clínicas de control prenatal realizadas a las embarazadas en el periodo del 01 de enero al 31 de marzo de 2016.

Para el desarrollo de la investigación se realizó un cálculo muestral ${ }^{19}$, teniendo en cuenta los siguientes factores: tamaño de la población, 1164; frecuencia esperada, 50\%; nivel de confianza, $95 \%$ y porcentaje de error del $4 \%$, lo que dio como resultado un tamaño muestral de 396 historias clínicas a auditar mediante la aplicación del instrumento.

La muestra por hospital quedó distribuida de la manera que se presenta en la Tabla 1.

Tabla 1. Distribución de la muestra según cada centro hospitalario.

\begin{tabular}{|c|c|c|}
\hline Nombre ESE & $\begin{array}{c}\text { Universo } \\
\text { de historias } \\
\text { clínicas }\end{array}$ & $\begin{array}{c}\text { No de histórias } \\
\text { clínicas } \\
\text { auditadas }\end{array}$ \\
\hline Hospital 1 & 154 & 53 \\
\hline Hospital 2 & 89 & 29 \\
\hline Hospital 3 & 378 & 131 \\
\hline Hospital 4 & 80 & 26 \\
\hline Hospital 5 & 206 & 71 \\
\hline Hospital 6 & 90 & 29 \\
\hline Hospital 7 & 167 & 57 \\
\hline Total & 1164 & 396 \\
\hline
\end{tabular}

Fuente: Cálculos de los autores.
Las historias clínicas fueron seleccionadas de manera aleatoria simple y secuencial dentro del total de las historias clínicas que cumplieron con los criterios de inclusión.

Los criterios de inclusión utilizados fueron los siguientes:

Primero, historias clínicas de pacientes con edad gestacional desde la semana 4 a la 38 de embarazo, en el periodo comprendido del 1 de enero al 30 de marzo del 2016.

Segundo, historias clínicas de gestantes que asistieron a las instituciones de salud a efectuar el control prenatal.

Tercero, historias clínicas de gestantes que asistieron a cita médica de revisión de exámenes generados durante su control prenatal.

\section{Variables y recolección de la información}

Treinta variables fueron tenidas en cuenta para el estudio, las cuales se distribuyeron en seis grupos de la siguiente manera: clasificación del riesgo obstétrico; controles y exámenes de laboratorio; historia clínica y carné materno perinatal; signos de alarma; provisión de micronutrientes; sistema de referencia y contrarreferencia.

Para la recolección de la información se utilizó como instrumento una lista de chequeo para evaluar la adherencia denominada: "Guía de control prenatal y factores de riesgo", cuyo autor es el Ministerio de Salud y Protección Social. Esta lista de chequeo fue adaptada por la Secretaría Seccional de Salud del Magdalena (Oficina de Calidad) con el fin de dar cumplimiento al objetivo de la circular conjunta externa 005 de febrero de $2012^{20}$. 
No se efectuó prueba piloto debido a que el instrumento de evaluación fue diseñado y validado por el Ministerio de Salud y Protección Social y adaptado por la Secretaría Seccional de Salud del Magdalena, además de ser utilizado trimestralmente para la evaluación periódica de la Adherencia a guías a las instituciones prestadoras de servicios de salud de la baja complejidad.

El instrumento evalúa la adherencia a las guías de atención por medio de las variables identificadas en las historias clínicas auditadas, y califica con 1 si cumple y 2, si no cumple con los ítems evaluados. Lo anterior permite obtener el porcentaje de cumplimiento por cada grupo, variable y hospital

El porcentaje de adherencia a las guías de control prenatal se estableció con base en los niveles definidos en la Tabla 2.

La recolección de los datos se llevó a cabo en cada una de las instituciones de salud en el periodo comprendido del 26 de julio al 23 de septiembre del 2016. Con el objetivo de preservar el anonimato de las instituciones hospitalarias se decidió enumerar los hospitales del 1 al 7.

Tabla 2. Porcentaje de Adherencia.

\begin{tabular}{|l|l|}
\hline \multicolumn{2}{|c|}{ Calificación por trimestre de edad gestacional evaluado en la historia clínica. } \\
\hline 95\% A 100\%: Óptimo & $\begin{array}{l}\text { Institución que cumple y requiere mantenimiento en la calidad } \\
\text { del control prenatal y por lo tanto tiene adherencia a la guía. }\end{array}$ \\
\hline 85\% A 94\%: Medio & $\begin{array}{l}\text { Institución que requiere mejorar y consolidar intervenciones } \\
\text { en los aspectos deficientes identificados en el control prenatal. }\end{array}$ \\
\hline Menos del 85\%: Deficiente & $\begin{array}{l}\text { La institución requiere intensificar el mejoramiento de los } \\
\text { aspectos deficientes identificados en el control prenatal. }\end{array}$ \\
\hline
\end{tabular}

Fuente: Ministerio de Salud y Protección Social

\section{Análisis estadístico}

El análisis se realizó mediante estadística descriptiva. Para ello, primero se consolidaron los datos en una matriz en Excel; segundo, se calcularon los porcentajes de cumplimiento para cada variable y grupo, y por último, se estableció el grado de adherencia a la guía por hospital y grupo de acuerdo a lo definido en tabla 2.

\section{Controles de sesgos}

Los controles de sesgos realizados en la investigación fueron los de selección y de clasificación ${ }^{21}$, ya que las historias clínicas estuvieron seleccionadas aleatoriamente dentro de la pobla- ción, por un periodo determinado y teniendo en cuenta criterios de inclusión; y a su vez clasificadas por cada institución de salud de los municipios que hacen parte de la subregión.

\section{Declaración sobre aspectos éticos}

Este estudio corresponde a una investigación sin riesgo, según el artículo 11 de la resolución 8430 de $1993^{22}$ del Ministerio de Salud, dado que el estudio fue realizado por medio de auditorías a historias clínicas y análisis de datos, preservando la información e integridad de los pacientes como lo menciona el artículo 11 de la resolución 839 de $2017^{23}$ y el artículo 10 de la ley 
1581 de $2012^{24}$. También cumple con los principios éticos contemplados en la declaración de Helsinki ${ }^{25}$ de la asociación médica mundial. El proyecto no fue sometido a un comité de ética e investigación debido a que no se divulgaron aspectos relacionados con nombres, número de documento o datos que puedan inducir a identificación del paciente; ni el instrumento utilizado presentaba dichos aspectos, la investiga- ción se limitó a verificar y auditar el contenido de las historias clínicas.

\section{RESULTADOS}

Se muestra a continuación en la Tabla 3 los resultados por cada uno de los centros hospitalarios estudiados.

Tabla 3. Resultado Total de la Adherencia a Guías de Control Prenatal en la subregión Norte del Departamento del Magdalena.

\begin{tabular}{|c|c|c|c|c|c|c|}
\hline $\begin{array}{l}\text { CLASE DE ITEM } \\
\text { EVALUADOR }\end{array}$ & $\begin{array}{c}\text { CENTRO } \\
\text { HOSPITALARIO }\end{array}$ & CUMPLE & NOCUMPLE & DEFICIENTE & MEDIO & OPTIMO \\
\hline \multirow{7}{*}{$\begin{array}{l}\text { Clasificación del } \\
\text { Riesgo Obstétrico }\end{array}$} & HOSPITAL 1 & $65 \%$ & $35 \%$ & $\mathrm{X}$ & & \\
\hline & HOSPITAL 2 & $100 \%$ & $0 \%$ & & & $\mathrm{X}$ \\
\hline & HOSPITAL 3 & $21 \%$ & $79 \%$ & $\mathrm{X}$ & & \\
\hline & HOSPITAL 4 & $25 \%$ & $75 \%$ & $\mathrm{X}$ & & \\
\hline & HOSPITAL 5 & $51 \%$ & $49 \%$ & $\mathrm{X}$ & & \\
\hline & HOSPITAL 6 & $65 \%$ & $35 \%$ & $\mathrm{X}$ & & \\
\hline & HOSPITAL 7 & $90 \%$ & $10 \%$ & & $\mathrm{X}$ & \\
\hline \multirow{7}{*}{$\begin{array}{l}\text { Controles y } \\
\text { exámenes de } \\
\text { laboratorio }\end{array}$} & HOSPITAL 1 & $69 \%$ & $31 \%$ & $\mathrm{X}$ & & \\
\hline & HOSPITAL 2 & $89 \%$ & $11 \%$ & & $\mathrm{X}$ & \\
\hline & HOSPITAL 3 & $64 \%$ & $36 \%$ & $\mathrm{X}$ & & \\
\hline & HOSPITAL 4 & $48 \%$ & $52 \%$ & $\mathrm{X}$ & & \\
\hline & HOSPITAL 5 & $62 \%$ & $38 \%$ & $\mathrm{X}$ & & \\
\hline & HOSPITAL 6 & $38 \%$ & $62 \%$ & $\mathrm{X}$ & & \\
\hline & HOSPITAL 7 & $71 \%$ & $29 \%$ & $\mathrm{X}$ & & \\
\hline \multirow{7}{*}{$\begin{array}{l}\text { Historia clínica y } \\
\text { Carné Materno } \\
\text { Perinatal }\end{array}$} & HOSPITAL 1 & $100 \%$ & $0 \%$ & & & $\mathrm{X}$ \\
\hline & HOSPITAL 2 & $100 \%$ & $0 \%$ & & & $\mathrm{X}$ \\
\hline & HOSPITAL 3 & $81 \%$ & $19 \%$ & $\mathrm{X}$ & & \\
\hline & HOSPITAL 4 & $100 \%$ & $0 \%$ & & & $\mathrm{X}$ \\
\hline & HOSPITAL 5 & $66 \%$ & $34 \%$ & $\mathrm{X}$ & & \\
\hline & HOSPITAL 6 & $82 \%$ & $18 \%$ & $\mathrm{X}$ & & \\
\hline & HOSPITAL 7 & $69 \%$ & $31 \%$ & $\mathrm{X}$ & & \\
\hline
\end{tabular}


Tabla 3. Continuación.

\begin{tabular}{|c|c|c|c|c|c|c|}
\hline $\begin{array}{l}\text { CLASE DE ITEM } \\
\text { EVALUADOR }\end{array}$ & $\begin{array}{c}\text { CENTRO } \\
\text { HOSPITALARIO }\end{array}$ & CUMPLE & NOCUMPLE & DEFICIENTE & MEDIO & OPTIMO \\
\hline \multirow{7}{*}{ Signos de alarma } & HOSPITAL 1 & $46 \%$ & $54 \%$ & $\mathrm{X}$ & & \\
\hline & HOSPITAL 2 & $29 \%$ & $71 \%$ & $\mathrm{X}$ & & \\
\hline & HOSPITAL 3 & $30 \%$ & $70 \%$ & $\mathrm{X}$ & & \\
\hline & HOSPITAL 4 & $8 \%$ & $92 \%$ & $\mathrm{X}$ & & \\
\hline & HOSPITAL 5 & $43 \%$ & $57 \%$ & $\mathrm{X}$ & & \\
\hline & HOSPITAL 6 & $92 \%$ & $8 \%$ & & $\mathrm{X}$ & \\
\hline & HOSPITAL 7 & $93 \%$ & $7 \%$ & & $\mathrm{X}$ & \\
\hline \multirow{7}{*}{$\begin{array}{l}\text { Provisión de } \\
\text { micronutrientes }\end{array}$} & HOSPITAL 1 & $100 \%$ & $0 \%$ & & & $\mathrm{X}$ \\
\hline & HOSPITAL 2 & $100 \%$ & $0 \%$ & & & $\mathrm{X}$ \\
\hline & HOSPITAL 3 & $81 \%$ & $19 \%$ & $\mathrm{X}$ & & \\
\hline & HOSPITAL 4 & $98 \%$ & $2 \%$ & & & $\mathrm{X}$ \\
\hline & HOSPITAL 5 & $100 \%$ & $0 \%$ & & & $\mathrm{X}$ \\
\hline & HOSPITAL 6 & $100 \%$ & $0 \%$ & & & $\mathrm{X}$ \\
\hline & HOSPITAL 7 & $100 \%$ & $0 \%$ & & & $\mathrm{X}$ \\
\hline \multirow{7}{*}{$\begin{array}{l}\text { Sistema de } \\
\text { Referencia y } \\
\text { Contrarreferencia }\end{array}$} & HOSPITAL 1 & $80 \%$ & $20 \%$ & $\mathrm{X}$ & & \\
\hline & HOSPITAL 2 & $100 \%$ & $0 \%$ & & & $\mathrm{X}$ \\
\hline & HOSPITAL 3 & $21 \%$ & $79 \%$ & $\mathrm{X}$ & & \\
\hline & HOSPITAL 4 & $25 \%$ & $75 \%$ & $\mathrm{X}$ & & \\
\hline & HOSPITAL 5 & $48 \%$ & $52 \%$ & $\mathrm{X}$ & & \\
\hline & HOSPITAL 6 & $55 \%$ & $45 \%$ & $\mathrm{X}$ & & \\
\hline & HOSPITAL 7 & $92 \%$ & $8 \%$ & & $\mathrm{X}$ & \\
\hline
\end{tabular}

Del total de los siete hospitales solo uno (14,2\%) cumple con la Clasificación del Riesgo obstétrico a las embarazadas en control prenatal, es decir que el médico identifica los factores de riesgo, evalúa los cambios y hace recomendaciones al respecto. Este hospital está en el 100\% de cumplimiento, y se ubica como óptimo en la semaforización de la tabla.

Con respecto al ítem de los controles y exámenes de laboratorio, se estableció que ningún hospital cumplió al 100\%, solo un hospital logró un 89\% ubicándolo en la semaforización de medio.
Con referencia al ítem de la Historia Clínica y Carné Materno Perinatal se determina que solo tres $(42,5 \%)$ hospitales cumplieron al 100\% óptimo.

En cuanto a los signos de alarma, se establece que un hospital $(28,5 \%)$ indaga este ítem con un promedio de $92,5 \%$, y se clasifica como medio.

En el ítem de provisión de micronutrientes se estableció que cinco $(71,4 \%)$ hospitales entregan a la gestante el Ácido Fólico y sulfato Ferroso como lo describe la guía de atención, es decir cumplen al 100\% óptimo. 
En el ítem de sistema de referencia y Contrarreferencia, se establece que dos $(28,5 \%)$ hospitales remiten a la embarazada a un nivel de complejidad mayor de acuerdo a la clasificación del riesgo obstétrico, de la categoría de óptimo.

Igualmente, se determinó que solo el hospital 2 cumple al $100 \%$ óptimo con cuatro $(66,6 \%)$ de los seis ítems evaluados (Clasificación del
Riesgo obstétrico, historia clínica materno y carné materno perinatal, provisión de micronutrientes y referencia y Contrarreferencia). Mientras que el resto de hospitales no cumple en ninguno de los ítems evaluados, por lo que puede considerarse que en la subregión norte del departamento del Magdalena hay baja adherencia a la Guía de Atención de detección de las alteraciones del embarazo, parto y puerperio de la Resolución 412 de $2000^{15}$.

Figura 1. Porcentaje de cumplimiento a las guías de atención clínica.

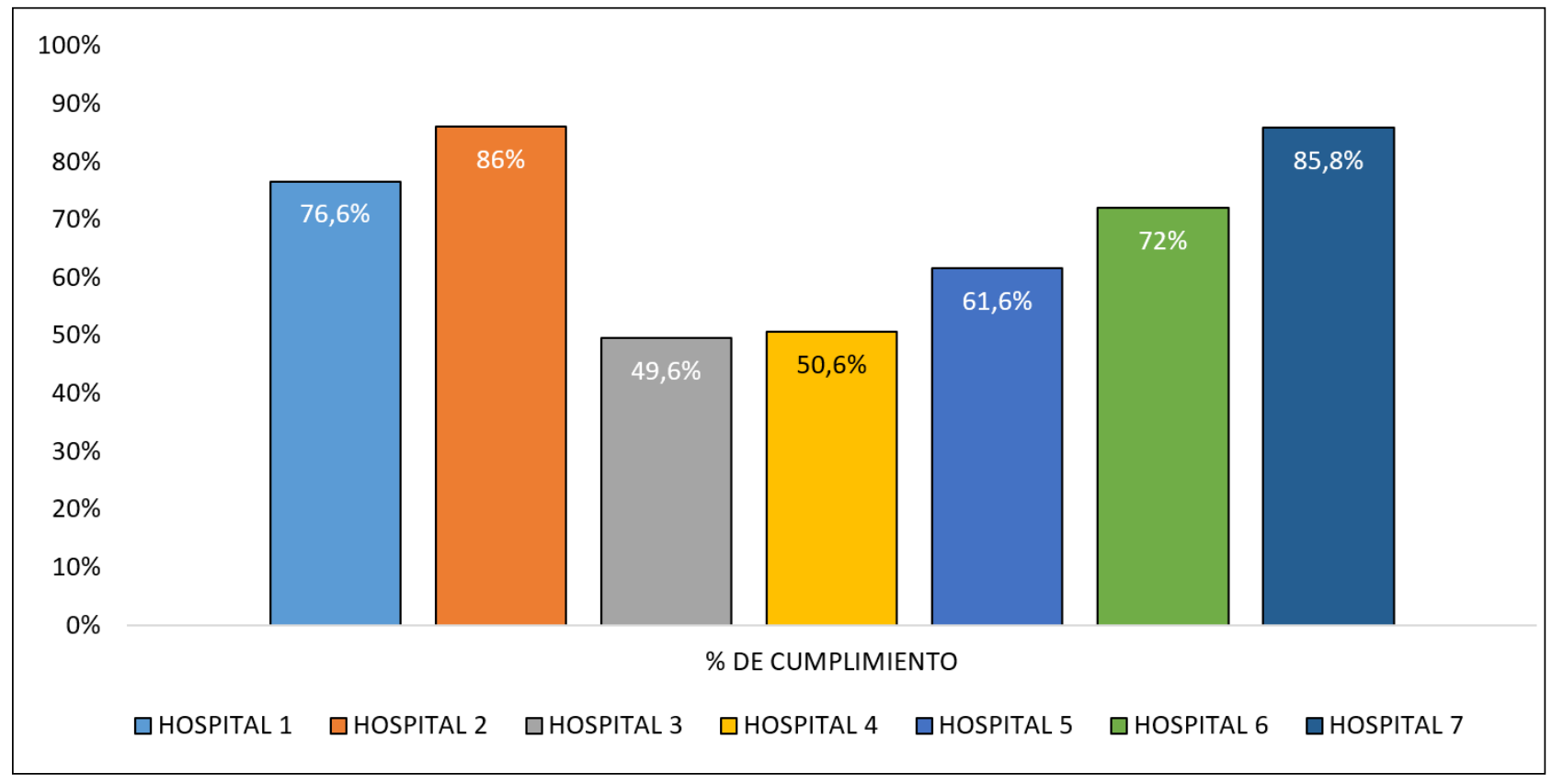

Fuente: Cálculos de los autores.

Como se observa en la Figura 1, el hospital 2 y el hospital 7 se encuentran con un porcentaje medio o aceptable de adherencia a las guías de atención Clínica en relación con los demás hospitales que tienen un porcentaje deficiente. Adicionalmente, se evidenciaron hallazgos como: diligenciamiento incompleto de las fichas CLAP (Centro Latino Americano de Perinatología) con un $72 \%$ de cumplimiento, en categoría deficiente; la curva de ganancia de peso y crecimiento uterino no se anotaban en cada uno de los controles; no se registra en la historia clínica y/o no se imparte la educación a la gestante; el ingreso al programa de Control Prenatal evidenciado en las distintas instituciones de salud iniciaba en el segundo y/o tercer trimestre de embarazo, lo cual supera ampliamente las recomendaciones de la OMS; no se cumple con los exámenes de laboratorio exigidos por la Guía de Control Prenatal y factores de riesgo de la Secretaria de Salud Distrital de Bogotá ${ }^{14,}$ en las instituciones de salud evaluadas. 
No existe buen manejo de los embarazos de alto riesgo, en las historias clínicas no se evidenciaba clasificación del embarazo según el riesgo, y no se realizaban las remisiones a un segundo nivel para un mejor manejo de estos. Los factores asociados a la baja adherencia fueron: el no cumplimiento de los protocolos de atención por parte de los profesionales de la salud, captación tardía de las gestantes, inicio de controles prenatales en el tercer trimestre de gestación, desconocimiento del embarazo por la gestante, falta de planificación familiar, dificultad en el traslado al lugar de atención, entre otros.

\section{DISCUSIÓN}

El control prenatal es una actividad de salud fundamental para garantizar el bienestar y la vida de la madre y el feto. Por ello se han desarrollado guías de atención que establecen los procesos que debe cumplir el equipo de salud durante la atención a las maternas. Para el caso colombiano, estas fueron establecidas en la resolución 412 del 2000 y sus anexos ${ }^{11}$.

El cumplimiento de lo definido en las guías de atención depende del grado de adherencia que a ella se tenga. Por tanto, la adherencia se convierte en elemento crítico para la obtención de resultados positivos en salud que beneficien a las maternas y a sus hijos en gestación ${ }^{16}$.

$\mathrm{Al}$ respecto, se encuentran en la literatura diversos estudios que analizan el grado de adherencia desde la perspectiva del paciente. Así, Rentería ${ }^{12}$ en una investigación adelantada en Cafesalud en San Juan IPS, evidenció que el factor que tuvo mayor impacto en la no adherencia a las guías de control prenatal fue educación brindada a la gestante.

Por otra parte, Rodríguez et $a l^{26}$ evidenciaron que los factores determinantes a la inasistencia a los controles prenatales estaban asociados en gran parte a barreras económicas, geográficas y socioculturales, las cuales no eran incluidas dentro de las estrategias y políticas de los sistemas de salud. Ante lo cual afirman que es necesario que la atención en salud goce de un enfoque inter y transectorial, donde el objetivo principal en la atención primaria en salud sea eliminar las barreras que limitan el acceso efectivo a los servicios de salud, en especial al control prenatal ${ }^{26,27}$.

En relación con la anterior investigación, Gaviria et $\mathrm{l}^{32}$ aseguran que uno de los factores que hacen que las gestantes inicien sus controles prenatales tardíamente son las barreras de acceso, entre estas la más evidente es la dificultad en el desplazamiento desde sus hogares al centro de atención; debido a esto las gestantes inician CPN en el tercer trimestre y, como consecuencia, presentan embarazos de alto riesgo y no se evidencia adherencia a las guías de control prenatal por parte de los centros de atención y/o los profesionales de la salud.

Villacis et $a l^{28}$ coinciden en afirmar la necesidad de que las gestantes que inician los controles prenatales durante el primer trimestre del embarazo logren que estos sean periódicos, completos e integrales, y reciban el apoyo interdisciplinario según el caso. En complemento, Martínez et $a l^{29}$ recomiendan que las intervenciones de los profesionales de enfermería estén reflejadas en incentivar y motivar a las gestantes a asistir oportunamente a los controles prenatales. Noguera y Dueñas ${ }^{30}$ consideran un factor determinante que las gestantes reciban educación y consejería durante los controles prenatales con el fin de que conozcan los signos y síntomas de alarma que se presentan durante el embarazo.

Ahora bien, es escasa la literatura que analiza la adherencia a las guías de control prenatal desde la perspectiva del equipo de salud ya 
que, aunque se realizan valoraciones de este tipo dentro de los procesos de evaluación de gerentes de las ESE, estas no son publicadas y quedan sus hallazgos restringidos al conocimiento de las juntas directivas y secretarías de salud territoriales. Por dicha razón, se dificulta poder comparar resultados y discutir posibles causas de los mismos desde una perspectiva académica.

Dentro de las pocas referencias que abordan la perspectiva de la adherencia por parte del equipo de salud, se menciona a Fraifer y García $^{31}$, quienes afirman que además de acompañar durante todo el proceso, no se debe olvidar que los controles prenatales ofrecen la oportunidad de contacto con el sistema de salud y, en muchos casos, se aprovecha para realizar intervenciones preventivas.

Es por ello que la presente investigación es relevante al presentar un análisis desde la perspectiva del prestador, gracias al cual se encuentra que ninguna de las instituciones de salud que conforman la subregión norte del departamento del Magdalena llegan al nivel óptimo de adherencia, y solo dos de ellas se ubican en nivel medio y las cinco restantes en deficiente.

Este hallazgo es preocupante si se tiene en cuenta que Colombia no logró cumplir con la meta esperada dentro de los compromisos de los ODM referente a mortalidad materna. Por ello, esta investigación puede servir para que las autoridades de salud del departamento definan estrategias encaminadas a mejorar la adherencia a la guía de atención prenatal.

\section{CONCLUSIÓN}

La adherencia a guías de atención de control prenatal por parte de las instituciones de salud públicas que conforman la subregión norte del departamento del Magdalena no es la deseada para garantizar la salud de las maternas y de sus hijos en gestación. Por ello, resulta imperioso que las autoridades del departamento realicen evaluaciones similares en las instituciones públicas de salud que conforman las demás subregiones con el fin de establecer si esta falencia en la adherencia se restringe a esta subregión o afecta a las demás.

Igualmente, sería deseable que se realicen investigaciones desde la perspectiva de las maternas para identificar qué factores contextuales y de infraestructura explicitan ellas como barreras de acceso que imposibilitan o retardan su atención en las instituciones públicas de salud de las subregiones en que se divide el departamento del Magdalena.

\section{DECLARACIÓN SOBRE CONFLICTOS DE INTERESES}

Los autores declaran no tener ningún conflicto de interés moral o económico.

\section{REFERENCIAS BIBLIOGRÁFICAS}

1. Organización Mundial de la Salud. Recomendaciones de la OMS sobre atención prenatal para una experiencia positiva del embarazo. Informe de un Grupo Científico de la OMS. Ginebra: OMS; 2016.

2. Burgos-Bernal G, Correa LF, Dávila CE, Gaviria-Uribe F, Girón- Vargas SL. Análisis de Situación en Salud (ASIS) Colombia 2016. Bogotá: Ministerio de Salud y Protección Social. Bogotá; 2016.

3. Gallego-Vélez L, Vélez- Álvarez GA, AgudeloJaramillo B. Panorama de la Mortalidad Materna. Modelos para el análisis de la mortalidad materna y perinatal. Medellín: Secretaría de Salud de Medellín. p 15-31; 2014. 
Adherencia a guías de atención de control prenatal en red pública de subregión norte, departamento del Magdalena (Colombia)

4. De la Hoz-Restrepo F, Gaviria-Uribe F, MuñozMuñoz NJ, Restrepo-Trujillo CI, Ruiz-Gómez F, Ospina-Martínez ML. Informe del evento Mortalidad Materna 2011.Bogotá: Primer informe observatorio nacional de salud 2011; 44-68.

5. Burgos-Bernal G, Gaviria-Uribe F, MuñozMuñoz NJ, Ruiz-Gómez F, Ospina-Martínez ML. Mortalidad Materna. Bogotá: Secretaría Distrital de Salud en Bogotá, Dirección de Salud Pública; 2013.

6. Gallego-Vega G. Una visión integral del modelo de seguridad clínica para la atención obstétrica. Medunab. 2015; 17(3):42-51

7. Álvarez-Toste M, Hinojosa-Álvarez M, Álvarez $\mathrm{S}$, López-Barroso R, González-Rodríguez $\mathrm{G}$, Carbonell I, et al. Morbilidad materna extremadamente grave, un problema actual. Rev Cubana Hig Epidemiol. 2011; 49(3):420-433.

8. Sánchez H, Pérez G, Pérez P, Vázquez F. Impacto del control prenatal en la morbilidad y mortalidad neonatal. Revista médica del instituto mexicano del seguro social. 2005; 43(5): 377-380.

9. Cancino E, León H, Otálora R, Pérez-González E, Sarmiento R, Yates A. Guía de control prenatal y factores de riesgo. Bogotá: Secretaría de Salud Distrital de Bogotá; 2015.

10. Álvarez- Bayona YA, Ascanio-Soto FA, BayonaÁlvarezE, Páez Pabón LD. Análisis dela captación tardía de las gestantes al primer control prenatal de la empresa social del estado Hospital Regional Noroccidental, IPS Abrego en el primer semestre del año 2014. Manizales: Universidad Católica de Manizales. Facultad de Posgrados; 2014.

11. Colombia. Ministerio de salud y Protección Social. Resolución 412 de 2000 por lo cual se establecen las actividades, procedimientos e intervenciones de demanda inducida y obligatorio cumplimiento y se adoptan las normas técnicas y guías de atención para el desarrollo de las acciones de protección específica y detección temprana y la atención de enfermedades de interés en salud pública. Diario oficial, 43956 (Marzo 31 2000).
12. Rentería-Córdoba SP.Evaluación dela adherencia a guías del programa de control prenatal en el Cafesalud (Cafi) San Juan IPS, primer semestre de 2010. Medellín: Universidad de CES Facultad de Salud Pública; 2010.

13. Pardo-Turriago R, Molano-Soto VM. Las guías de práctica clínica: una herramienta de participación en la construcción de una política pública. Acta Neurol Colomb 2014; 30(4):307-313.

14. Duarte-Osorio A, Sánchez-Díaz N. Plan de implementación de dos Guías de Práctica Clínica (GPC) contenidas en las Guías de Atención Integral (GAI) en el Sistema General de Seguridad Social en Salud de Colombia: A) "Detección temprana del episodio depresivo y trastorno depresivo recurrente en adultos. Atención integral de los adultos con diagnóstico de episodio depresivo o trastorno depresivo recurrente (GPCdepresión)".B) "Detección temprana, diagnóstico y tratamiento de la fase aguda de intoxicación de pacientes mayores de 18 años con abuso o dependencia del alcohol (GPC-alcohol)". Rev Colomb Psiquiatr 2012; 41(4): 826-841.

15. Múnera HD, Piedrahita-Calderón ME. Evaluación de la baja adherencia a guías médicas implementadas en la ESE hospital San Vicente de Paúl de Caldas Antioquia primer semestre 2008. Medellín: Universidad de Antioquia, Facultad de Salud Pública; 2008.

16. Pinilla-Ferro R, Coy-Torres Y, Jiménez-Barbosa W. Grado de adherencia a las guías clínicas de defectos refractivos por parte de los optómetras en la consulta externa de optometría en una IPS, primer semestre del 2012. Ciencia Y Tecnología Para La Salud Visual Y Ocular [serial on the Internet]. (2013, Julio), [citado en Mayo 7, 2018]; 11(2): 67.

17. Documento Programa Territorial de Reorganización, rediseño y modernización de las Empresas Sociales del Estado del Departamento del Magdalena. Magdalena: Secretaría Seccional de Salud del Magdalena; 2011: 8-9. 
Katty Johana Ortiz-Grisolle, Kelly Garcia-Galezo, Angela Patricia Gómez-Cardona, Sindy Julieth Maestre-Álvarez, Graciela Emilia Vargas-Guerrero, Néstor Ramos-Navas, Wilson Giovanni Jiménez-Barbosa

18. Colombia. Ministerio de Salud y Protección Social. Resolución 743 de 2013 por la cual se modifica la Resolución 710 de 2012 y se dictan otras disposiciones. Diario Oficial, 48737 (Marzo 192013).

19. Aguilar-Barojas S. Fórmulas para el cálculo de la muestra en investigaciones de salud. Tabasco, México: Salud en Tabasco; 2005.

20. 2Colombia. Ministerio de Salud y Protección Social. Circular conjunta 005 por la cual se promueve e impulsa de forma conjunta acciones preventivas que propendan por el eficiente cumplimiento de la normatividad vigente para la intensificación de acciones para garantizar la maternidad segura a nivel nacional. Diario Oficial, 48374 (Marzo 16 2012).

21. Del Pino-Casado R, Frías-Osuna A, PalominoMoral PA. El control de sesgos en la investigación cuantitativa enfermera. Revista iberoamericana de enfermería comunitaria 2011; 4(1): 24-34.

22. Colombia. Ministerio de Salud y Protección Social. Resolución 8430 de 1993 por la cual se establecen las normas científicas, técnicas y administrativas para la investigación en salud. Diario Oficial, 39120 (Dic 19 1993).

23. Colombia. Ministerio de Salud y Protección Social. Resolución 839 de 2017 Por la cual se modifica la Resolución 1995 de 1999 y se dictan otras disposiciones. Diario Oficial, 000839 (Marzo 24 2017).

24. Cárdenas-Santamaría M, Correa-Palacio LS, Diazgranados-Guida S, Molano-Vega D, SantosCalderón JM. Ley estatutaria 1581/2012 de 17 de octubre. Bogotá: Congreso de Colombia; 2012.

25. Mazzanti-Di Ruggiero, M. Declaración de Helsinki, principio y valores bioéticos en juego en la investigación con seres humanos. Revista colombiana de bioética. 2011; 6(1):125-144.

26. Rodríguez-Páez FG, Jiménez-Barbosa WG, Jiménez-González CA, Coral-Córdoba AE,
Ramírez- Solano PC, Ramos-Navas NR. Efecto de las barreras de acceso sobre la asistencia a citas de programa de control prenatal y desenlaces perinatales. Rev Gerenc Polít Salud. 2014; 1 (27): 206-221.

27. Noel Karin. Factores asociados al abandono del control prenatal en el centro de salud los libertadores, distrito de San Martin de Porres. Marzo a Mayo de 2016, Lima, Perú. Perú: Universidad de San Martin de Porres, Facultad de Obstetricia y Enfermería; 2016.

28. Villacis-Vallejos C, Becerra-Cornejo D, Negrete Kerguelen L. Adherencia al control prenatal en la clínica de gestantes adolescentes del hospital de Engativá de Bogotá. Bogotá: Universidad Nacional de Colombia, Departamento de Obstetricia y Ginecología; 2012.

29. Martínez-Suarez AM, Mayorga-Camargo VL, Quevedo-Moreno KS. Adherencia al control prenatal, dificultades y retos. Bogotá: Pontificia Universidad Javeriana, Facultad de enfermería; 2012.

30. Noguera AM, Dueñas MC. Evaluación de la calidad de la atención del programa de control prenatal a partir de la atención brindada a las usuarias en el centro de salud suroccidente de la ese Popayán enero de 2011 - junio de 2011. Popayán: Universidad de Caldas, Facultad de posgrados; 2011.

31. Fraifer S, García M. Control prenatal en embarazadas de bajo riesgo I. Rev Evid Actual Practicas ambulatorias, 2004; 7(6): 173-177.

32. Gaviria-Uribe A, Ruiz- Gómez F, Muñoz- Muñoz NJ, Burgos-Bernal G, Arias-Duarte JF, García S. Guía Técnica "Buenas prácticas para la seguridad del paciente en la atención en salud". Garantizar la atención segura de la gestante y el recién nacido. Bogotá: Secretaria de Salud Distrital de Bogotá; 2010. 